Bulletin of Mathematical Biology (1999) 61, 1019-1030

Article No. bulm.1999.0128

Available online at http://www.idealibrary.com on IDE $\mathbf{A}$.

\title{
Membrane Skeleton Detachment in Spherical and Cylindrical Microexovesicles
}

\author{
HENRY HÄGERSTRAND \\ Department of Biology, \\ Åbo Akademi University, \\ FIN-20520, \\ Åbo/Turku, \\ Finland
}

VERONIKA KRALJ-IGLIČ

Institute of Biophysics,

Medical Faculty, University of Ljubljana,

SI-1000 Ljubljana, Slovenia

\section{MALGORZATA BOBROWSKA-HÄGERSTRAND}

Department of Biology,

Åbo Akademi University,

FIN-20520,

Åbo/Turku,

Finland

ALEŠ IGLIČ*

Laboratory of Applied Physics,

Faculty of Electrical Engineering, University of Ljubljana,

SI-1000 Ljubljana, Slovenia

E-mail: ales.iglic@fe.uni-lj.si

\begin{abstract}
We observed that amphiphile-induced microexovesicles may be spherical or cylindrical, depending on the species of the added amphiphile. The spherical microexovesicle corresponds to an extreme local difference between the two monolayer areas of the membrane segment with a fixed area, while the cylindrical microexovesicle corresponds to an extreme local area difference if the area of the budding segment is increased due to lateral influx of anisotropic membrane constituents. Protein analysis showed that both types of vesicles are highly depleted in the membrane skeleton. It is suggested that a partial detachment of the skeleton in the budding region is favoured due to accumulated skeleton shear deformations in this region.
\end{abstract}

(C) 1999 Society for Mathematical Biology

\footnotetext{
*Author to whom correspondence should be addressed: Dr Aleš Iglič, Faculty of Electrical Engineering, Tržaška 25, SI-1000 Ljubljana, Slovenia.

0092-8240/99/061019+ $12 \quad \$ 30.00 / 0$

(C) 1999 Society for Mathematical Biology
} 


\section{INTRODUCTION}

A variety of treatments with water-soluble amphiphilic compounds can induce a release of exovesicles from human red blood cells (RBCs) [see Bütikofer et al. (1987), Hägerstrand and Isomaa (1989)]. The shape of these vesicles is usually spherical but is also sometimes cylindrical (Hägerstrand and Isomaa, 1992). It was found that RBC vesicles are usually depleted in major components of the membrane skeleton (Allan et al., 1976; Wagner et al., 1986; Hägerstrand and Isomaa, 1994; Knowles et al., 1997). This depletion may be due to a disruption of the skeleton (Kozlov et al., 1990) or its detachment from the membrane prior to vesiculation (Liu et al., 1989; Iglič et al., 1995; Knowles et al., 1997; Bobrowska-Hägerstrand et al., 1998). In addition, the RBC microexovesicles may be depleted in the skeleton if the budding region is located above the gap in the skeleton network (Kozlov et al., 1990; Saxton, 1992).

The redistributions of other membrane components, i.e., membrane lipids and integral membrane proteins, during the vesiculation process (Allan et al., 1976; Discher et al., 1994; Hägerstrand and Isomaa, 1994; Knowles et al., 1997) may play an important role in stabilization of the microexovesicle shape (Lipowsky, 1993; Kralj-Iglič et al., 1996; Seifert, 1997; Kralj-Iglič et al., 1999).

The features observed in vesiculation of RBCs may be also relevant for understanding the vesiculation processes in some other cells and organelles (Wagner $e t$ al., 1986). It is therefore of great interest to understand the mechanism taking place in RBC membrane budding and vesiculation involving also the skeleton depletion in RBC microexovesicles. The aim of the present work is to elucidate possible physical mechanisms leading to the formation of membrane skeleton depleted RBC cylindrical or spherical microexovesicles.

\section{Materials AND Methods}

2.1. Chemicals. Dodecyltrimethylammonium bromide (C12-TAB) was purchased from Sigma and dodecyl D-maltoside (C12-MALT) from Fluka.

2.2. Erythrocytes. Blood was drawn from the authors by vein puncture into heparinized tubes. The erythrocytes were washed three times in a buffer containing $145 \mathrm{mM} \mathrm{NaCl}, 5 \mathrm{mM} \mathrm{KCl}, 4 \mathrm{mM} \mathrm{Na} \mathrm{HPO}_{4}, 1 \mathrm{mM} \mathrm{NaH} \mathrm{PO}_{4}, 1 \mathrm{mM} \mathrm{MgSO}$, $1 \mathrm{mM} \mathrm{CaCl}_{2}$ and $10 \mathrm{mM}$ glucose (pH 7.4). The erythrocytes were then suspended in the buffer at a cell density of $1.65 \cdot 10^{9}$ cells $/ \mathrm{ml}$ and kept at $4{ }^{\circ} \mathrm{C}$ until used. The blood was used within $30 \mathrm{~h}$ after drawing.

2.3. Incubation of erythrocytes. Aliquots of a prewarmed $\left(37^{\circ} \mathrm{C}\right)$ erythrocyte stock suspension were pipetted into polystyrene tubes or glass vials containing a prewarmed $\left(37^{\circ} \mathrm{C}\right)$ buffer with amphiphiles. The final cell density was $1.65 \cdot 10^{8}$ 
cells $/ \mathrm{ml}$ (about $1.5 \%$ haematocrit) and the incubations were carried out in a shaking thermostatted bath at $37^{\circ} \mathrm{C}$. The amphiphiles were used at sublytic concentrations determined as previously described (Isomaa et al., 1987).

2.4. Isolation of erythrocytes and exovesicles. Erythrocytes were isolated and processed as previously described (Hägerstrand and Isomaa, 1989, 1992, 1994). In short, following incubation with C12-TAB $(300 \mu \mathrm{M})$ or C12-MALT $(40 \mu \mathrm{M})$ for $60 \mathrm{~min}$ at $37^{\circ} \mathrm{C}$, erythrocytes were pelleted by centrifugation at $1.4 \cdot 10^{3} \mathrm{~g}$ for $10 \mathrm{~min}$. Exovesicles were, after an additional centrifugation of the supernatant as above, pelleted by centrifugation of the resulting supernatant at $2 \cdot 10^{4} \mathrm{~g}$ for $40 \mathrm{~min}$.

\subsection{Processing of erythrocytes and microexovesicles for transmission electron} microscopy (TEM). Following isolation, erythrocytes and microexovesicles were fixed and post-fixed in $1 \%$ glutaraldehyde and osmiumtetroxide, respectively, in a buffer for $30 \mathrm{~min}$ at room temperature. After dehydration in a graded series of acetone/water, the samples were embedded in Epon. Thin sections were stained with lead citrate and post-stained with uranyl acetate.

2.6. SDS-PAGE for polypeptide and glycoprotein separation. Sodium dodecyl sulphate polyacrylamide gel electrophoresis (SDS-PAGE) was performed in a Bio-Rad Minigel apparatus using a 5\% polyacrylamide stacking gel and a 7.5\% polyacrylamide separation gel, according to Laemmli (1970). Samples were prepared by adding a Laemmli sample buffer to packed mother cells or to pelleted exovesicles. The polypeptide bands were visualized with Coomassie brilliant blue staining.

\section{EXPERimental Results}

Predominantly spherical microexovesicles were released to the outer solution by C12-TAB [Fig. 1(a)], while C12-MALT induced the release of mainly cylindrical microexovesicles [Fig. 1(b)]. The spherical microexovesicles had a diameter of about $150 \mathrm{~nm}$. The length of the cylindrical microexovesicles was often 400$850 \mathrm{~nm}$ and their diameter was about $80 \mathrm{~nm}$. Invaginated stomatocyte-like spherical microexovesicles were frequently seen [Fig. 1(a)]. The spherical microexovesicles frequently had a tail- or tongue-like protrusion.

We have tested a large number of echinocytogenic amphiphiles and showed that only two anisotropic amphiphiles, i.e., the dimeric amphiphile dioctyldiQAS (Hägerstrand and Isomaa, 1992) and C12-MALT with a bulky dimeric polar head induce a release of predominantly cylindrical microexovesicles [Fig. 1(b)], while all other tested echinocytogenic amphiphiles induce a release of predominantly spherical microexovesicles (Hägerstrand and Isomaa, 1992, 1994). 

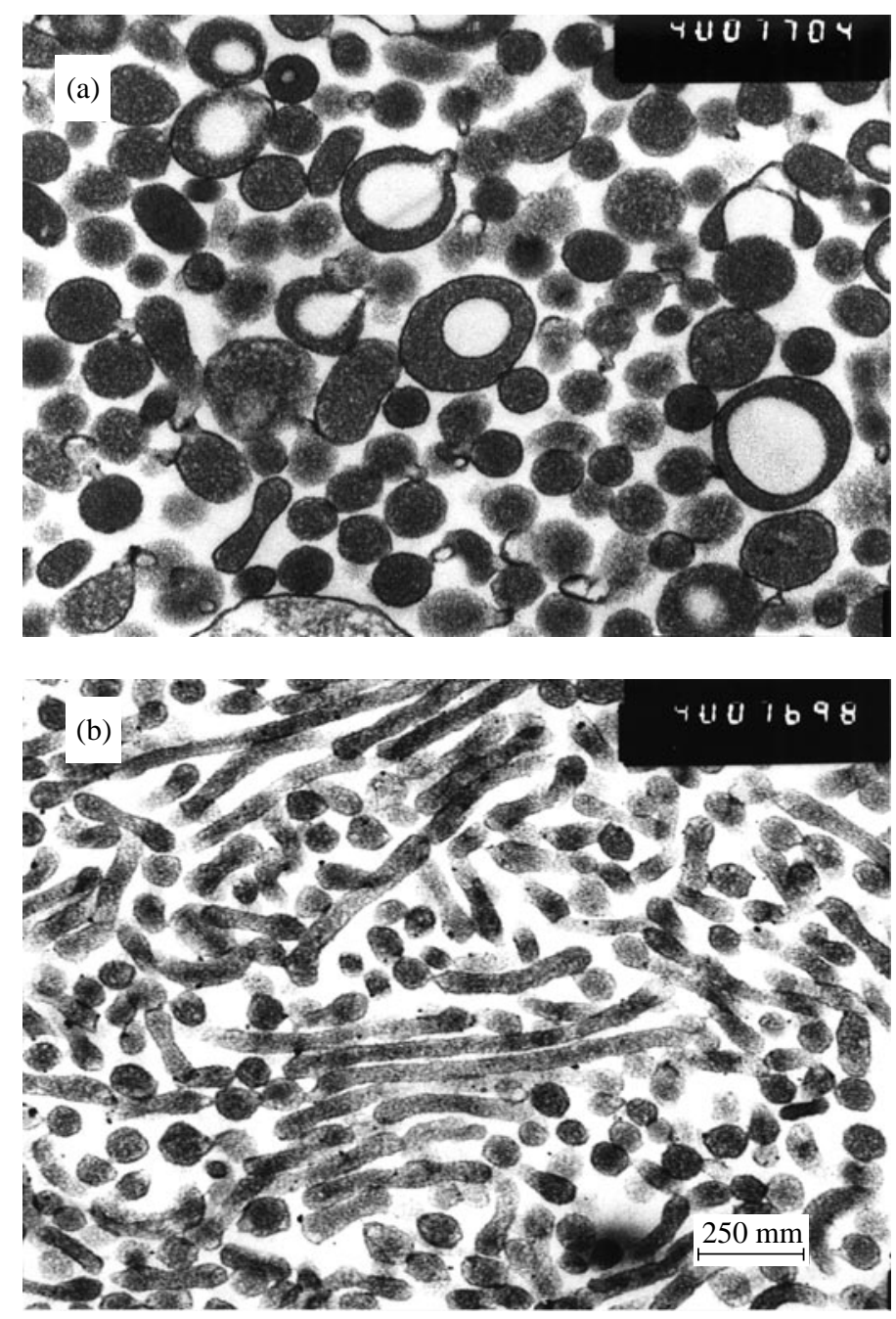

Figure 1. TEM micrographs showing released microexovesicles following amphiphile treatement of human erythrocytes. Human erythrocytes were incubated $\left(5 \mathrm{~min}, 37^{\circ} \mathrm{C}\right)$ with (a) C12-TAB $(300 \mu \mathrm{M})$ or (b) C12-MALT $(40 \mu \mathrm{M})$. 


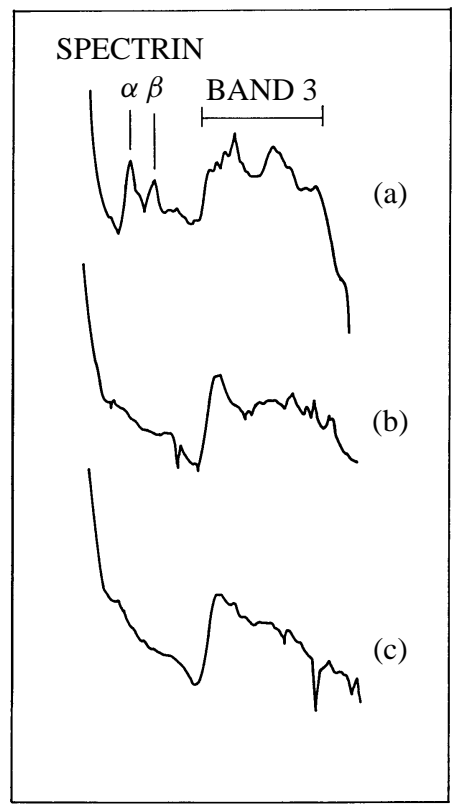

Figure 2. Polypeptide profiles of human erythrocytes treated (30 min, $37^{\circ} \mathrm{C}$ ) with amphiphiles and of released microexovesicles, following SDSPAGE and Coomassie brilliant blue staining. (a) Membrane of the parent erythrocyte treated with C12-MALT, (b) microexovesicles released following treatment with C12-MALT and (c) microexovesicles released following treatment with $\mathrm{C} 12-\mathrm{TAB}$.

While the membrane of the amphiphile-treated parent erythrocytes showed the presence of $\alpha$-spectrin and $\beta$-spectrin [Fig. 2(a)], the released spherical and cylindrical microexovesicles were depleted in, or lacked, $\alpha$-spectrin and $\beta$-spectrin [Fig. 2(b) and (c)]. Band 3 protein, however, was also present in the microexovesicles.

\section{Theoretical Predictions}

4.1. Shapes of the membrane segment corresponding to extreme area difference $\Delta \boldsymbol{A}$. The microvesiculation process is thought to be induced by an elevation of the local difference between the outer and the inner monolayer areas of the membrane bilayer $\triangle A$ (Wiese et al., 1992; Bukman et al., 1996; Iglič and Hägerstrand, 1999). An increase of the local area difference of the membrane segment during the budding process $\triangle A$ can be achieved in many different ways. For example, by intercalation of the amphiphilic molecules from the outer solution in the outer bilayer leaflet of the cell membrane (Sheetz and Singer, 1974; Svetina et al., 1982; Iglič and Hägerstrand, 1999), due to conformational changes of membrane proteins (Gimsa and Ried, 1995), or due to a lateral flow of membrane constituent molecules 
to the segment from the surrounding membrane (Israelachvili et al., 1976). The equilibrium $\triangle A$ and shape of the segment at a given external condition are determined by the minimum of the total free energy of the system (Israelachvili $e t$ al., 1976; Wiese et al., 1992; Iglič et al., 1998) including the bending and relative stretching energy of the segment (Bukman et al., 1996), the shear energy (Evans and Skalak, 1980; Waugh, 1996; Iglič, 1997) and the energy due to inhomogeneous distributions of membrane components (Andelman et al., 1992; Lipowsky, 1993; Mohandas and Evans, 1994; Kralj-Iglič et al., 1996). However, it has been shown recently that the final shape of $\mathrm{RBC}$ microexovesicles is determined by the condition of the local extreme $\triangle A$ (Iglič and Hägerstrand, 1999). In order to obtain the shapes of the membrane segment having an extreme area difference $\triangle A$ at a given area of the segment, a variational problem is stated by constructing a functional (Iglič and Hägerstrand, 1999)

$$
G=\triangle A-\lambda_{A} \cdot\left(\int \mathrm{d} A-A\right),
$$

where $\triangle A$ for thin membranes is

$$
\triangle A=h \int\left(C_{1}+C_{2}\right) \mathrm{d} A,
$$

$h$ is the distance between the neutral surfaces of the outer and the inner membrane lipid leaflet, $\lambda_{A}$ is the Lagrange multiplier, $\mathrm{d} A$ is the area element and $C_{1}$ and $C_{2}$ are the principal membrane curvatures.

The analysis is restricted to axisymmetric shapes. It is chosen so that the symmetry axis of the body coincides with the $x$-axis, in order that the shape of the segment be given by the rotation of the function $y(x)$ around the $x$-axis, In this case the principal curvatures are expressed by $y(x)$ and its derivatives with respect to $x ; y^{\prime}=\frac{\partial y}{\partial x}$ and $y^{\prime \prime}=\frac{\partial^{2} y}{\partial x^{2}}$, as

$$
C_{1}=\frac{1}{y \sqrt{1+y^{\prime 2}}}
$$

and

$$
C_{2}=-\frac{y^{\prime \prime}}{\left(\sqrt{1+y^{\prime 2}}\right)^{3}} .
$$

The area element is $\mathrm{d} A=2 \pi \sqrt{1+y^{\prime 2}} y \mathrm{~d} x$.

Inserting the above expressions for $C_{1}, C_{2}$ and $\mathrm{d} A$ into equation (1) and rearranging the terms, the functional normalized with respect to $2 \pi h$ is expressed as

$$
\begin{gathered}
G=\int g\left(x, y, y^{\prime}, y^{\prime \prime}\right) \mathrm{d} x, \\
g\left(x, y, y^{\prime}, y^{\prime \prime}\right)=1-\frac{y y^{\prime \prime}}{1+y^{\prime 2}}-2 \lambda_{A} y \sqrt{1+y^{\prime 2}},
\end{gathered}
$$


where $\lambda_{A} / h \rightarrow \lambda_{A}$.

The variation $\delta G=0$ is performed by solving the Euler-Poisson equation

$$
\frac{\partial g}{\partial y}-\frac{\mathrm{d}}{\mathrm{d} x}\left(\frac{\partial g}{\partial y^{\prime}}\right)+\frac{\mathrm{d}^{2}}{\mathrm{~d} x^{2}}\left(\frac{\partial g}{\partial y^{\prime \prime}}\right)=0 .
$$

Obtaining the necessary differentiation of equation (7), the Euler-Poisson equation can be written as

$$
\frac{2 y^{\prime \prime}}{\left(1+y^{\prime 2}\right)^{2}}+\lambda_{A}\left(\frac{1}{\sqrt{1+y^{\prime 2}}}-\frac{y y^{\prime \prime}}{\left(\sqrt{1+y^{\prime 2}}\right)^{3}}\right)=0 .
$$

If the area of the segment is fixed $\left(\lambda_{A} \neq 0\right)$, there is an analytical solution of equation (8), given by a circle of radius $r_{\mathrm{s}}$ :

$$
y=\sqrt{r_{\mathrm{s}}^{2}-x^{2}}
$$

which represents a sphere of a radius $1 / r_{\mathrm{s}}=\lambda_{A}$ and a segment of a plane $1 / r_{\mathrm{s}}=0$. If the area $A$ is not fixed, i.e., if $\lambda_{A}=0$, the possible analytical solution of the equation is $y=$ const, representing a cylinder. A boundary condition should be stated, such as a requirement for a fixed radius of the cylinder.

For $\lambda_{A}=0$ we have chosen the cylindrical solution of equation (7) which is consistent with recent theoretical predictions (Fournier, 1996; Kralj-Iglič et al., 1999) and with experimental results indicating that anisotropic amphiphilic molecules have the capacity to induce predominantly cylindrical microexovesicles [Fig. 1(b)]. Namely, it has been shown recently that the orientational ordering of anisotropic molecules embedded in the membrane may favour a thin cylindrical shape in order to minimize the membrane free energy (Fournier, 1996; Kralj-Iglič et al., 1999).

On the basis of the results presented it can be concluded that the final shape of the exovesicles corresponding to the extreme value of the segment area difference $\triangle A$ can be spherical or cylindrical. The exovesicle is spherical if the area of the budding segment $A$ is fixed during the budding process (Iglič and Hägerstrand, 1999) and cylindrical if the area of the budding segment A increases during the budding due to the influx of the anisotropic membrane constituents (Israelachvili et al., 1976; Kralj-Iglič et al., 1999) to the region of the segment.

\subsection{A possible role of membrane shear elasticity in depletion of membrane} skeleton in red blood cell exovesicles. It has been shown that the area of the isolated RBC membrane skeleton is, at physiological conditions, smaller than the area of the RBC (Lange et al., 1982; Svoboda et al., 1992). This implies that in the intact cell the skeleton is expanded by means of its interactions with the membrane bilayer (Steck, 1989) to completely underlay the inner surface of the bilayer. We 
have shown (Iglič et al., 1995; Bobrowska-Hägerstrand et al., 1998) that a partial detachment of the membrane skeleton in the budding region of the cell membrane may be energetically favourable if the decrease of the skeleton expansion energy after partial skeleton detachment is larger than the corresponding increase of the bilayer-skeleton binding energy. However, in our previous work, the role of shear deformations of the skeleton in the budding region was not considered. Therefore, in this section, the increase of the shear energy of the skeleton during the budding process is estimated.

Recently, a new constitutive model for the membrane skeleton behaviour was suggested which takes into account that the membrane skeleton is locally compressible (Mohandas and Evans, 1994; Discher and Mohandas, 1996). However, due to simplicity in this work, the shear energy of the skeleton is calculated using an approximate expression (Evans and Skalak, 1980):

$$
W=\frac{\mu}{2} \int\left(\lambda_{\mathrm{m}}^{2}+\lambda_{\mathrm{m}}^{-2}-2\right) \mathrm{d} A,
$$

where the membrane skeleton is considered laterally incompressible (Waugh, 1996), $\mu$ is the membrane skeleton area shear modulus, $\lambda_{\mathrm{m}}$ is the principal extension ratio along the meridional direction (Iglič, 1997) and d $A$ is the membrane area element which is chosen sufficiently small so that we may consider it approximately flat. It has been indicated that in spite of oversimplifications made by using equation (10), the corresponding calculated shear energy of the skeleton may still be realistic (Waugh, 1996).

The shapes of the cylindrical and spherical protrusions, leading to the formation of cylindrical and spherical exovesicles, respectively, are described by two simple geometrical models with two parameters. In the case of cylindrical protrusions these two parameters are the length of the cylindrical part of the protrusion $l$ and the radius $r$ [Fig. 3(a)]. The shape of the spherical protrusion is determined by the radius of the vesicle $R$ and the angle $\vartheta$ determining the width of the vesicle neck [Fig. 3(b)].

The shear energy of the cylindrical protrusion [Fig. 3(a)] can be calculated according to the method of Evans and Skalak (1980) as described in detail elsewhere (Iglič, 1997):

$$
\begin{aligned}
W_{\mathrm{c}} / \mu= & 2 \pi r^{2}\left(\ln 2-\frac{5}{8}\right)+\pi l^{2}+\frac{\pi}{2} r^{2} \ln \left(1+\frac{l}{r}\right)+\left(2 \pi r l+2 \pi^{2} r^{2}-4 \pi r^{2}\right) \\
& \ln \left(\frac{l}{2 r}+\frac{\pi}{2}\right)+2 \pi \int_{0}^{\pi / 2}\left(\frac{1}{2}\left(\lambda_{\mathrm{m}}^{2}+\lambda_{\mathrm{m}}^{-2}-2\right)(r+r(1-\cos \omega))\right) r \mathrm{~d} \omega
\end{aligned}
$$

where

$$
\lambda_{\mathrm{m}}^{2}=\frac{2 \pi r^{2}(1-\sin \omega)+2 \pi r l+4 \pi r^{2} \omega}{\pi(2 r-r \cos \omega)^{2}} .
$$


(a)

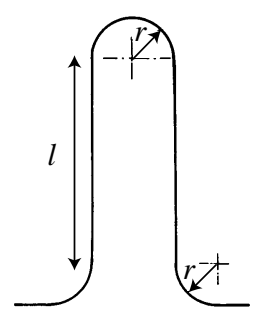

(b)

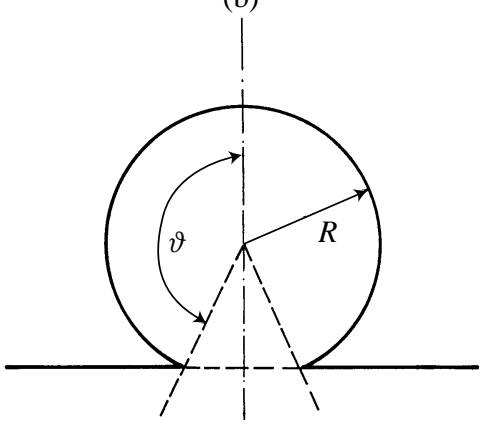

Figure 3. The parameters characterizing the geometrical model of (a) cylindrical and (b) spherical protrusions of the red blood cell membrane.

The last integral in equation (11) is obtained numerically using the trapezoidal formula (Iglič, 1997).

Using the same method (Evans and Skalak, 1980; Iglič, 1997), the shear energy of the spherical protrusion [Fig. 3(b)] can be calculated as:

$$
\begin{aligned}
W_{\mathrm{s}} / \mu= & 2 \pi R^{2}\left(\ln \frac{2}{(1+\cos \vartheta)}+\frac{3}{4} \cos \vartheta-\frac{1}{8} \cos ^{2} \vartheta-\frac{5}{8}\right) \\
& +\pi R^{2}\left(\sin ^{2} \vartheta+(1-\cos \vartheta)^{2}\right) \cdot \ln \sqrt{1+\frac{\sin ^{2} \vartheta+(1-\cos \vartheta)^{2}}{\sin ^{2} \vartheta}}
\end{aligned}
$$

While calculating the shear energies $W_{\mathrm{c}}$ and $W_{\mathrm{s}}$, the flat membrane is considered as an initial reference state, where it is assumed that in the reference state the shear energy is zero (Evans and Skalak, 1980; Iglič, 1997).

Figure 4(a) shows the shear energy of the cylindrical protrusion as a function of the length of its cylindrical part $l$. Figure 4(b) shows the dependence of the shear energy of the spherical protrusion on the angle $\vartheta$ [Fig. 3(b)] determining the size of the protrusion. It can be seen in Fig. 4 that the shear energy of the protrusion strongly increases in the budding process for both the cylindrical as well as for the spherical shape of the protrusion. On the basis of the results given in Fig. 4 it can be concluded that a partial detachment of the skeleton from the bilayer in the budding region of the red blood cell membrane is energetically favourable, since in this way the accumulated shear deformations in the protrusions can be relaxed.

\section{Conclusions}

We observed that the shape of amphiphile-induced microexovesicles is spherical or cylindrical, depending on the species of the added amphiphile. The spherical 

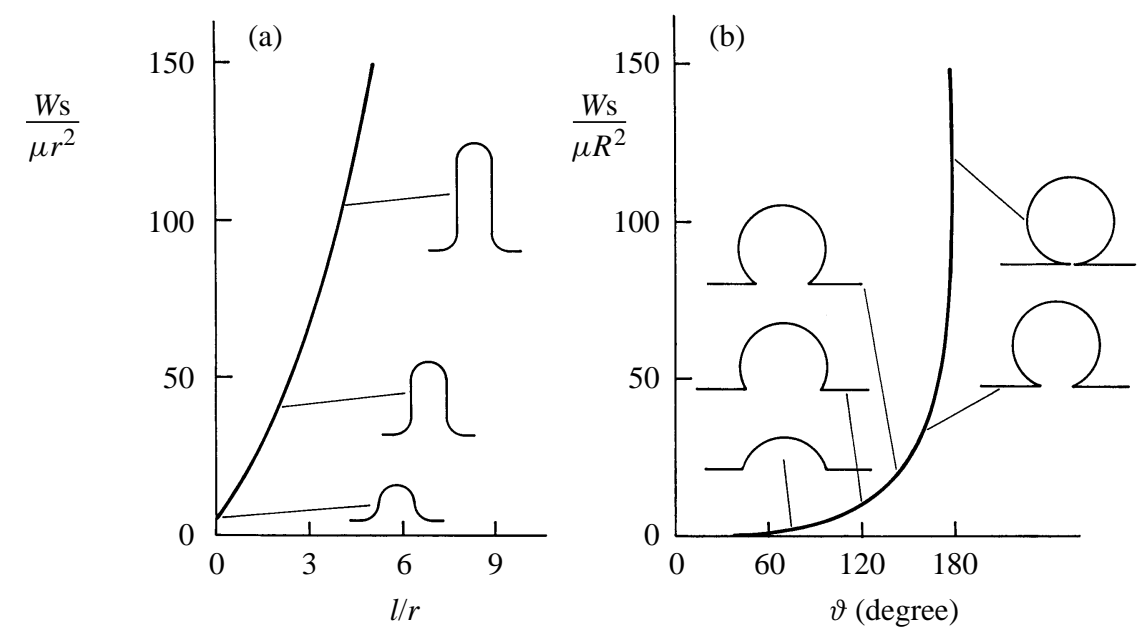

Figure 4. Relative shear energy of (a) cylindrical protrusion as a function of the length of its cylindrical part and (b) of spherical protrusion as a function of the width of the protrusion neck.

microexovesicle corresponds to the extreme difference between the two monolayer areas $\triangle A$ of the budding segment with the fixed area (Iglič and Hägerstrand, 1999). It has been shown here that the cylindrical microexovesicle corresponds to the extreme local area difference $\triangle A$ assuming that the area of the budding segment is increased due to the influx of the anisotropic membrane constituents in the budding region.

In order to predict whether the budding will lead to a spherical or to a cylindrical protrusion/vesicle, it should be established which of the two processes is possible. If both of them are possible it should be distinguished which of them would be energetically more favourable. Therefore, the free energy of the budding segment under consideration (Israelachvili et al., 1976; Wiese et al., 1992; Bukman et al., 1996; Boulbitch, 1998; Kralj-Iglič et al., 1999) should be minimized, taking into account the local composition of the segment. This is, however, beyond the scope of this work.

In conclusion, we have shown by protein analysis that spherical as well as cylindrical microexovesicles are depleted in $\alpha$-spectrin and $\beta$-spectrin. We propose here that this depletion is mainly due to accumulated shear deformations in the budding membrane region which are expected to relax when the skeleton is detached.

\section{REFERENCES}

Allan, D., M. M. Billah, J. B. Finean and R. H. Michell (1976). Release of diacylglycerolenriched vesicles from erythrocytes with increased intracelular $\left[\mathrm{Ca}^{2+}\right]$. Nature 261, 5860. 
Andelman, D., T. Kawakatsu and K. Kawasaki (1992). Equilibrium shape of twocomponent unilamellar membranes and vesicles. Europhys. Lett. 19, 57-62.

Bobrowska-Hägerstrand, M., H. Hägerstrand and A. Iglič (1998). Membrane skeleton and red blood cell vesiculation at low pH. Biochim. Biophys. Acta 1371, 123-128.

Boulbitch, A. A. (1998). Deflection of a cell membrane under application of local force. Phys. Rev. E 57, 1-5.

Bukman, D. J., J. H. Yao and M. Wortis (1996). Stability of cylindrical vesicles under axial tension. Phys. Rev. E 54, 5463-5468.

Bütikofer, P., U. Brodbeck and P. Ott (1987). Modulation of erythrocyte vesiculation by amphiphilic drugs. Biochim. Biophys. Acta 901, 291-295.

Discher, D. E. and N. Mohandas (1996). Kinematics of red cell aspiration by fluorescenceimaged microdeformation. Biophys. J. 71, 1680-1694.

Discher, D. E., N. Mohandas and E. A. Evans (1994). Molecular maps of red cell deformation: hidden elasticity and in situ connectivity. Science 266, 1032-1035.

Evans, E. and R. Skalak (1980). Mechanics and Thermodynamics of Biomembranes, Boca Raton, FL: CRC Press.

Fournier, J. B. (1996). Nontopological saddle-splay and curvature instabilities from anisotropic membrane inclusions. Phys. Rev. Lett. 76, 4436-4439.

Gimsa, J. and C. Ried (1995). Do band 3 protein conformational changes mediate shape changes of human erythrocytes. Mol. Membr. Biol. 12, 247-254.

Hägerstrand, H. and B. Isomaa (1989). Vesiculation induced by amphiphiles in erythrocytes. Biochim. Biophys. Acta 982, 179-186.

Hägerstrand, H. and B. Isomaa (1992). Morphological characterization of exovesicles and endovesicles released from human erythrocytes following treatment with amphiphiles. Biochim. Biophys. Acta 1109, 117-126.

Hägerstrand, H. and B. Isomaa (1994). Lipid and protein composition of exovesicles released from human erythrocytes following treatment with amphiphiles. Biochim. Biophys. Acta 1190, 409-415.

Iglič, A. (1997). A possible mechanism determining the stability of spiculated red blood cells. J. Biomech. 30, 35-40.

Iglič, A. and H. Hägerstrand (1999). Amphiphile induced spherical microexovesicle corresponds to an extreme local area difference between two monolayers of the membrane bilayer. Med. Biol. Eng. Comput. 37, 125-129.

Iglič, A., V. Kralj-Iglič and H. Hägerstrand (1998). Amphiphile induced echinocytespheroechinocyte red blood cell shape transformation. Eur. Biophys. J. 27, 335-339.

Iglič, A., S. Svetina and B. Žekš (1995). Depletion of membrane skeleton in red blood cell vesicles. Biophys. J. 69, 274-279.

Isomaa, B., H. Hägerstrand and G. Paatero (1987). Shape transformations induced by amphiphiles in erythrocytes. Biochim. Biophys. Acta 899, 93-103.

Israelachvili, J. N., D. J. Mitchell and B. W. Ninham (1976). Theory of self-assembly of hydrocarbon amphiphiles into micelles and bilayers. J. Chem. Soc. Faraday Trans. 72, 1525-1568. 
Knowles, D. W., L. Tilley, N. Mohandas and J. A. Chasis (1997). Erythrocyte membrane vesiculation: model for the molecular mechanism of protein sorting. Proc. Natl. Acad. Sci. USA 94, 12696-12974.

Kozlov, M. M., L. V. Chernomordik and V. S. Markin (1990). A mechanism of formation of protein-free regions in the red cell membrane: the rupture of the membrane skeleton. J. Theor. Biol. 144, 347-365.

Kralj-Iglič, V., V. Heinrich, S. Svetina and B. Žekš (1999). Free energy of closed membrane with anisotropic inclusions. Eur. Phys. J. B 10, 5-8.

Kralj-Iglič, V., S. Svetina and B. Žekš (1996). Shapes of bilayer vesicles with membrane embedded molecules. Eur. Biophys. J. 24, 311-321.

Laemmli, U. K. (1970). Cleavage of structural proteins during the assembly of the head of bacteriophage T4. Nature 227, 680-685.

Lange, Y., R. A. Hadesman and T. L. Steck (1982). Role of reticulum in the stability and shape of the isolated human erythrocyte membrane. J. Cell Biol. 92, 714-721.

Lipowsky, R. (1993). Domain induced budding of fluid membranes. Biophys. J. 64, 11331138.

Liu, S. C., L. H. Derick, M. A. Duquette and J. Palek (1989). Separation of the lipid bilayer from the membrane skeleton during discocyte-echinocyte transformation of human erythrocyte ghosts. Eur. J. Cell Biol. 49, 358-365.

Mohandas, N. and E. Evans (1994). Mechanical properties of the red cell membrane in relation to molecular structure and genetic defects. Ann. Rev. Biophys. Biomol. Struct. 23, 787-818.

Saxton, M. J. (1992). Gaps in the erythrocyte membrane skeleton: a stretched net model. J. Theor. Biol. 155, 517-536.

Seifert, U. (1997). Configurations of fluid membranes and vesicles. Adv. Phys. 46, 13-137.

Sheetz, M. P. and S. J. Singer (1974). Biological membranes as bilayer couples. A molecular mechanism of drug-erythrocyte interactions. Proc. Natl. Acad. Sci. 71, 4457-4461.

Steck, T. L. (1989). Red cell shape, in Cell Shape: Determinants, Regulation and Regulatory Role, W. Stein and F. Bronner (Eds), New York: Academic Press, pp. 205-246.

Svetina, S., A. Ottova-Leitmannova and R. Glaser (1982). Membrane bending energy in relation to bilayer couples concept of red blood cell shape transformations. J. Theor. Biol. 94, 13-23.

Svoboda, K., C. F. Schmidt, D. Branton and S. M. Block (1992). Conformation and elasticity of the isolated red blood cell membrane skeleton. Biophys. J. 63, 784-793.

Wagner, G. M., D. T. Y. Chiu, M. C. Yee and B. H. Lubin (1986). Red cell vesiculation-a common membrane physiological event. J. Lab. Clin. Invest. 108, 315-324.

Waugh, R. E. (1996). Elastic energy of curvature-driven bump formation on red blood cell membrane. Biophys. J. 70, 1027-1035.

Wiese, W., W. Harbich and W. Helfrich (1992). Budding of lipid bilayer vesicles and flat membranes. J. Phys. Condens. Matter 4, 1647-1657. 